\title{
Pure NOTES Transvesical \\ Venous Ligation: Translational Animal Model of Varicocelectomy
}

\author{
Luís Osório, David Silva, Riccardo Autorino, Rocco Damiano, Jorge Correia-Pinto, and \\ Estêvão Lima
}

OBJECTIVE $\quad$ To assess the feasibility of pure natural orifice transluminal endoscopic surgery (NOTES) transvesical venous ligation mimicking bilateral varicocelectomy in an animal model.

MATERIALS AND Transvesical NOTES bilateral venous ligation was performed in 6 female pigs by considering METHODS lower epigastric vessels as a model for gonadal vessels. Under flexible cystoscopic guidance, a cystotomy was created on the anterior bladder. The flexible cystoscope was introduced through the over tube, and the lower epigastric vessels were visualized in retroflexion. Thulium laser was used to cut and coagulate the vessels. A bladder catheter was left in place for 4 days in all animals and they were sacrificed 15 days after the procedure.

RESULTS The procedure was successfully carried out in all animals without intraoperative complications. Epigastric vessels were safely cut and coagulated using the thulium laser. Median time for the overall procedure, including establishment of the transvesical port, was 23 minutes (range 20-30). No complications were encountered during the postoperative follow-up period. Postmortem examination revealed complete coagulation and separation of vessels.

CONCLUSION An animal model mimicking a NOTES transvesical bilateral varicocelectomy procedure is successfully shown in the present study. Despite being encouraging, these novel findings need to be interpreted with caution. Further research is warranted and development of purpose-built instrumentation is awaited to define potential urological applications of transvesical NOTES. UROLOGY 78: 1082-1088, 2011. (C) 2011 Elsevier Inc.

$\mathrm{V}$ aricocele represents the most common correctable etiology found in adult men with infertility. ${ }^{1}$ In the last 20 years minimally invasive treatment options for varicocele, such as antegrade sclerotherapy and microsurgical and laparoscopic varicocele ligation have been popularized and have gained growing acceptance. $^{2-4}$

Among other surgical specialties, urology could be considered the most "minimally invasive." Since the early 1990s, the shift toward less invasive procedures has

Financial Disclosure: Jorge Correia-Pinto is consultant/adviser for Karl Storz (Tuttlingen, Germany).

Funding Support: Support for this study was provided by Grants PTDC/SAU-OSM/ 105578/2008 from FCT-Portugal and Karl Storz (Tuttlingen, Germany).

From the Surgical Sciences Research Domain, Life and Health Sciences Research Institute (ICVS), School of Health Sciences, University of Minho, Braga, Portugal; Department of Medicine, Centro Hospitalar do Médio Ave, Famalicão, Portugal; Urology Clinic, Second University of Naples, Italy; Urology Unit, Magna Graecia University, Catanzaro, Italy; Department of Pediatric Surgery, Hospital São João, Porto, Portugal; and Dept of Urology, Hospital de Braga, Braga, Portugal

Reprint requests: Estevao Lima, M.D., Ph.D., F.E.B.U., Surgical Sciences Research Domain-Life and Health Sciences Research Institute (ICVS), School of Health Sciences, University of Minho, Campus de Gualtar, 4709-057 Braga, Portugal. E-mail: estevaolima@ecsaude.uminho.pt

Submitted for publication: February 3, 2011, accepted (with revisions): May 28, 2011 progressively made endoscopy and laparoscopy the first choice for the majority of urological interventions, overcoming open surgery. ${ }^{5}$

In 2004, Kalloo et al described the feasibility of accessing the abdominal cavity by performing endoscopy through a transgastric approach, giving birth to the concept of natural orifice transluminal endoscopic surgery (NOTES). ${ }^{6}$ NOTES eliminates the need for abdominal incisions, theoretically resulting in decreased pain, faster convalescence, improved cosmesis, and absence of wound infections and hernias. Since that pioneering report, the concept of performing surgery through natural body orifices has been recognized and investigated. ${ }^{7}$

Each access site for NOTES carries individual advantages and pitfalls and, after the initial enthusiasm, many limitations surfaced and jeopardized application in humans. In 2006, the bladder was proposed by Lima as an attractive alternative option for NOTES access, breaking through a classical sanctuary of urology-the wall of the urinary tract. ${ }^{8}$

Initial surviving experimental studies have demonstrated the feasibility of an atraumatic method to create a transvesical port, ${ }^{8}$ the possibility to reach the thoracic cavity, ${ }^{9}$ the usefulness in a combined access (transvesical 
and transgastric) to perform cholecystectomy ${ }^{10}$ and nephrectomy, ${ }^{11}$ and, more recently, the safety of endoscopic closure of bladder perforation. ${ }^{12}$ Thus, transvesical NOTES access has been adopted only to perform procedures on the upper abdomen and thorax and to assist transgastric procedures for combined NOTES.

The aim of the current study was to assess the feasibility of pure NOTES transvesical venous ligation mimicking bilateral varicocelectomy in an animal model.

\section{MATERIAL AND METHODS}

\section{Study Protocol}

This was a survival study done in six $35-45-\mathrm{kg}$ female pigs (Sus scrofa domesticus) after approval by the ethical review board of Minho University, Braga, Portugal. The animals were withdrawn from food for 24 hours and from water for 6 hours before the surgical procedure. Each animal underwent transvesical NOTES bilateral varicocelectomy by considering lower epigastric vessels as a model for gonadal vessels. All procedures were performed using general anesthesia, as described previously. ${ }^{9}$ Pigs were followed after surgery for 15 days before sacrifice and necropsy examination.

\section{Surgical Technique}

A 9.5-Fr ureteroscope (27 002 L, Karl Storz, Tuttlingen, Germany) was introduced through the urogenital sinus and urethra into the bladder with $\mathrm{CO}_{2}$ distension. The cystotomy site was selected on the bladder dome. A mucosal incision was made with scissors (27 $424 \mathrm{U}$, Karl Storz) introduced in the working channel of the ureteroscope. Subsequently, a 5-Fr open-ended ureteral catheter (Selectip, Angiomed GmbH, Baden-Württemberg, Germany) was pushed forward through the incision into the peritoneal cavity. Guided by a 0.035 -inch flexible-tip guidewire $\left(\mathrm{RF}^{*} \mathrm{GA} 35153 \mathrm{M}\right.$ Terumo Medical Corporation, São Paulo, Brazil), the cystotomy was enlarged with the dilator of a 250-105 ureteroscope sheath (Boston Microvasive, Boston Scientific, Mason, $\mathrm{OH}$ ), which was enveloped with a $5.5-\mathrm{mm}$ overtube. Then, a 16-Fr flexible video cystourethroscope (11 272VP, Karl Storz) was introduced into the peritoneal cavity through the overtube and allowed to create pressure-controlled $\mathrm{CO}_{2}$ pneumoperitoneum up to $12 \mathrm{~mm} \mathrm{Hg}$ using an insufflator (Endoflator, Karl Storz). With the video cystourethroscope in retroflexion, exploration of the upper abdomen and pelvis was performed. Once the internal inguinal ring was identified, lower epigastric vessels were cut and coagulated by using a thulium laser (RevoLix, Lisa Laser Products, Katlenburg-Lindau, Germany). In all animals the procedure was repeated on the contralateral side. The video cystourethroscope was removed and the peritoneal cavity was decompressed through the overtube. At the end of the procedure we observed the inner bladder wall morphology and a 14-Fr Foley catheter was inserted into the bladder.

\section{Postoperative Care}

At the end of the surgical intervention, all animals received $1.2 \mathrm{~g}$ amoxicillin and clavulanic acid intravenously. Oral feeding was started within the following 24 hours. The bladder catheter was removed 4 days after surgery. The animals were closely monitored for any signs of postoperative complications, distress, behavior changes, and anorexia or weight loss. They were evaluated daily and followed for 15 days. Animals were sacrificed and necropsy examination was then performed, with particular attention to the vesical incision site and lower epigastric vessels.

\section{RESULTS}

Cut and coagulation of the lower epigastric vessels with thulium laser by the pure NOTES transvesical approach was successfully performed in all 6 pigs. The surgical steps to create the transvesical port (cystoscopy, bladder mucosal incision, cystotomy, and transvesical overtube passage) were easily and rapidly performed without complications.

Video cystourethroscope was used most of the time in retroflexion, allowing an easy visualization of the anterior abdominal wall and lower epigastric vessels bilaterally in all the procedures (Figure 1A). The introduction of the thulium laser fiber through the working channel did not encounter any difficulty during the procedure and the cutting and the coagulation of the vessels was safely performed under visual control (Fig. 1B, C) without hemorrhage in all animals. Thulium laser vessels ligation was clean and rapid, with excellent hemostasis. Withdrawing the fiber tip away from the tissue allowed coagulation of larger vessels and achieved complete hemostasis. The largest vessel coagulate was $4.2 \mathrm{~mm}$ in diameter.

Median time for the overall procedure, including establishment of the transvesical port, was 23 minutes (range 20-30). Procedure time decreased with experience. Smoke did not appear to be a limitation of the technique, because vessel ligation was quickly achieved in all animals. Intraoperative blood loss was insignificant. At necropsy, exploration of the entire peritoneal cavity, anterior abdominal wall, and bladder showed complete healing of the bladder wall incision, no visceral injury, and complete coagulation and separation of the lower epigastric vessels (Fig. 2).

\section{COMMENT}

Most of the current surgical approaches to varicocele are based on interruption of pathologic venous drainage route of the testis, with ligation or occlusion of the internal spermatic veins. Although providing an alternative to the open technique, the laparoscopic approach carries higher costs, being therefore considered appropriate only for bilateral varicocele. ${ }^{13}$ Although less invasive, angiographic occlusion appears to have higher failure rates. ${ }^{14}$ Recently, Kaouk et al presented their initial experience in 3 children undergoing single-port laparoscopic left varicocelectomy. ${ }^{15}$

NOTES represents an appealing concept to pursue in minimally invasive procedures and it is currently in its early stages of development in visceral surgery. ${ }^{6,16}$

To bring it closer to the routine clinical setting, the ideal way to access the peritoneal cavity through a natural orifice remains to be determined. Certainly each site 


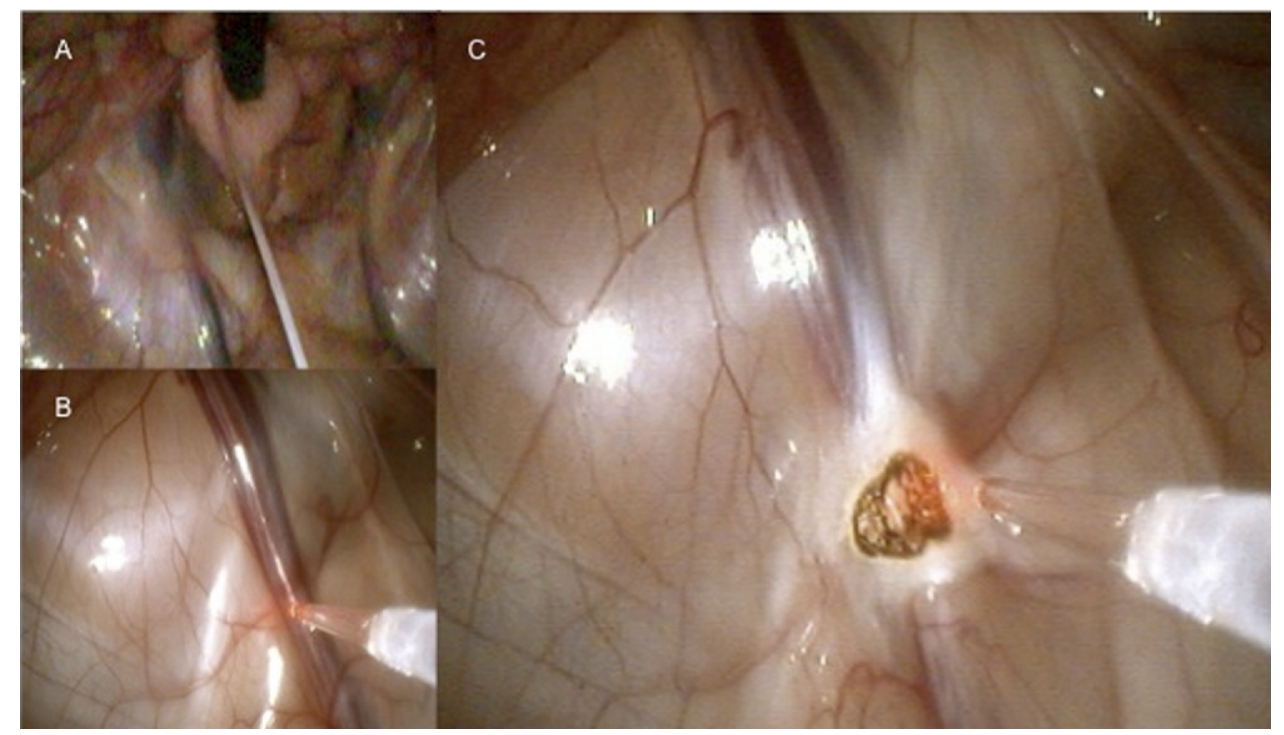

Figure 1. (A) Flexible cystoscope view of epigastric vessels bilaterally in retroflexion. (B) Thulium laser performed under visual control. (C) Clean and rapid cutting with excellent hemostasis.

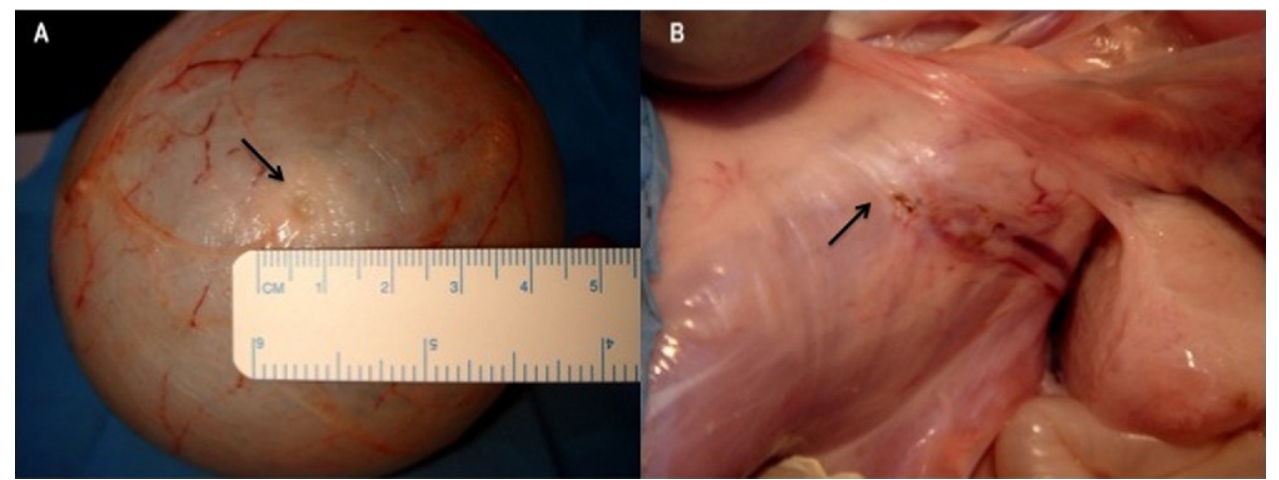

Figure 2. Necropsy images from the bladder and epigastric vessels. (A) Outside view showing complete healing of the vesicotomy. (B) Coagulation and separation of the epigastric vessels.

has individual advantages and pitfalls and after the initial enthusiasm, many limitations surfaced and jeopardized application in humans.

The first historically applied access for NOTES- the transgastric approach ${ }^{17}$ - presents some restrictions. The per-oral route is not sterile and certainly bacteria will gain access to the peritoneal cavity. The views of the upper abdominal organs, such as the liver and the gallbladder, are limited; these organs require retroflexion for the endoscope, as opposed to views of pelvic organs that are in the direct line of vision of a transgastric endoscope. ${ }^{18}$ The optimal site of the gastrotomy to provide safe entry has to be elucidated. Reliable endoscopic gastric closure technique must be developed and improved. ${ }^{19}$

As all other cavities were explored, the first conclusion we made was that lower abdominal ports might be better for upper abdominal procedures and useful as an accessory port for transgastric access.
The transvaginal approach seems to be the most reliable for human application at this time. Secure closure can be readily attained using the standard surgical technique, and several procedures have been described in the gynecologic literature of using the vagina to access the peritoneal cavity with low complication rate. ${ }^{20,21}$ The vagina has also been described as a route for specimen extraction. This has fostered the translation of NOTES transvaginal access to the clinical arena with the recent description of pure NOTES transvaginal nephrectomy in humans. ${ }^{22}$ The major limitation of the transvaginal route is that it can be used only in female patients. Moreover, sexual function and quality of life issues remain to be determined. ${ }^{23}$

The transcolonic approach allows good access to the peritoneal cavity, mainly for the upper abdomen, but the risk of peritoneal infection is high and refinements in colonic preparation, luminal sterilization, and closure technique will be required before translation to human. ${ }^{24}$ 
In 2006, Lima et al were the first to introduce the bladder as a portal for NOTES. ${ }^{8}$ In 2007, the same group reported on a combined transvesical and transgastric porcine cholecystectomy and concluded that transvesical access facilitated visualization and dissection during cholecystectomy. ${ }^{10}$ In the same year, Gettman and coworkers, after preliminary cadaveric studies, applied transvesical NOTES in the clinical setting by performing, without complications, a transvesical peritoneoscopy on a man before a robot-assisted prostatectomy. ${ }^{25}$

Transvesical access offers an appealing access route to the peritoneal cavity, overcoming almost all of the drawbacks of other routes. Cystotomy usually heals spontaneously by keeping the transurethral catheter in place. ${ }^{8}$ The urinary tract is normally sterile, thus in theory the risk of infections and intraperitoneal or retroperitoneal contamination should be minimized. Recently, McGee and colleagues examined the resultant microbial contamination of the human peritoneum after transvesical incision and exposure of the abdomen to bladder contents during robot-assisted laparoscopic prostatectomy. The authors concluded that prolonged peritoneal exposure to bladder contents demonstrates minimal contamination of the abdominal cavity and is without postoperative infectious significance. $^{26}$

Bladder location, in the most anterior portion of the pelvic cavity, enables easy peritoneal access above bowel loops, theoretically minimizing the risk of complications. This approach also offers a visualization of all the intraperitoneal structures with a direct line of sight; they offer an en face orientation of the upper abdominal organs, allowing better visualization and the ability to work straightforwardly. Spatial orientation is easier than that offered by the per-oral or transcolonic approach. Although lower abdominal ports are better for the upper abdominal organs approach and vice versa, and the pelvic cavity can be easily approached through the dependent direction, we did not find major difficulties because most of the procedure is performed in retroflexion. Manual deflection of the flexible cystoscope, a technique well known to most urologists, permitted precise control of laser fiber for vessel coagulation.

Laparoscopic surgery requires sufficient dissection devices in terms of safe and fast tissue cutting and vessel closure. Thulium lasers have a $2-\mu \mathrm{m}$ wavelength that matches the water absorption peak, allowing efficient tissue cutting with limited thermal damage. The main advantage of the thulium laser might be that time-consuming instrumentation changes are reduced, increasing working speed because significantly less bleeding occurs or it could be handled by the laser itself. The optimum condition for use of this laser is submersion in a low flow of saline or water, because in a dry environment there is an excessive generation of smoke, with poor visualization of the tissue. Ex vivo studies suggest that irrigation could help to reduce smoke during laparoscopic surgery. ${ }^{27}$ Vessel coagula- tion was quickly achieved in our experience and this did not appear to be a limitation of the procedure. In 2007, Theisen and colleagues confirmed that small $(<0.5 \mathrm{~mm})$ and larger $(1-2.3 \mathrm{~mm})$ vessels were successfully occluded, with the $2-\mu \mathrm{m}$ laser scalpel, up to $89-100 \%$ and $65-74 \%$ respectively. ${ }^{28}$ In general, coagulation is obtained by contraction of the vessel wall caused by the thermally induced shrinkage of surrounding tissue and formation of an endoluminal coagulum. Regarding the potential benefits of this method to cut and coagulate vessels endoscopically, we believe that it may be possible to avoid the need of ligation or clipping. Bilateral lower epigastric vessels coagulation in a porcine model was intentionally chosen because it anatomically mimics a current procedure performed in human males-laparoscopic varicocelectomy. Furthermore, these vessels have similar caliber to human gonadal vessels near the entrance of the inguinal ring. In our experience, coagulation was easily achieved under visual control without hemorrhage in all the animals.

Despite these potential advantages, several issues remain to be answered. Would it be possible to easily handle and perform procedures through the transvesical port in a human model? Attempting to answer this question, Branco et al recently reported that peritoneoscopy and liver biopsy using a rigid ureteroscope through a transvesical port is feasible in a human male cadaver. ${ }^{29}$ For bilateral varicocelectomy, one of the major problems of this route may be the length of the male urethra, because the flexible video cystourethroscope size is short. Probably, in the near future, biomedical engineering will need to develop longer scopes and instruments. Will it be possible to use thulium laser to coagulate a vessel as large as a dilated human gonadal vein that can be larger than $3 \mathrm{~mm}$ ? Thulium laser seems to be a promising hemostatic device for high-precision dissection and coagulation during interventions via the NOTES approach. Boylu et al evaluated the feasibility of NOTES transgastric partial nephrectomy with a nonischemic approach in a porcine model. ${ }^{30}$ After incision of Gerota's fascia, the left kidney upper pole was excised using the thulium laser without hilar dissection or clamping. Although steering the laser fiber with the gastroscope deflection was challenging, they confirmed that thulium laser was able to cut the tissue and simultaneously coagulate the vessels successfully.

Finally, there were limitations in the present study. Albeit effective in mimicking a varicocelectomy procedure, the animal model adopted herein carries its own drawbacks. A division of the epigastric vessels was performed instead of a real bilateral varicocelectomy. The adopted model was created by using female pigs. It would have been interesting to use male pigs to evaluate a spermatic cord and to decipher spermatic veins vs artery and vas deferens. However, male pigs were not used 
because of anatomical limitation due to the length of the male pig urethra, because the length of the flexible cystourethroscope would have been insufficient. Therefore, it remains to determine whether the challenges of dividing the epigastric arteries in a female pig are the same as the challenges that would be encountered with managing the testicular artery veins and vas deferens in a male model. Despite the successful occlusion of epigastric vessels by using laser, suitability of the laser for occluding spermatic veins remains to be demonstrated. Again, the challenges that could be encountered during a real varicocelectomy procedure, where fine dissection between structures to be sectioned and those to be spared, are not addressed in this experimental model. New instrumentation is expected to aid in further development of this field of research.

While looking forward to specialized suturing devices that should be developed for further NOTES applications, we provide initial evidence that bilateral varicocelectomy by applying thulium laser might represent a simple intraabdominal surgical procedure with low hemorrhagic risk. However, much study is still needed to refine techniques, verify safety, and document efficacy before translation into humans to minimize unexpected complications.

\section{CONCLUSION}

An animal model mimicking a NOTES transvesical bilateral varicocelectomy procedure by using thulium laser is successfully shown in the present study. Despite encouraging results, these novel findings need to be interpreted with caution, and further research using the transvesical approach is warranted. Development of purpose-built platforms and devices are awaited to expand the potential urological applications of NOTES.

\section{References}

1. Goldstein M. Adolescent varicocele. J Urol. 1995;153:484-485.

2. Barroso U Jr., Andrade DM, Novaes H, et al. Surgical treatment of varicocele in children with open and laparoscopic Palomo technique: a systematic review of the literature. J Urol. 2009;181:27242728 .

3. May M, Johannsen M, Beutner S, et al. Laparoscopic surgery versus antegrade scrotal sclerotherapy: retrospective comparison of two different approaches for varicocele treatment. Eur Urol. 2006;49: 384-387.

4. Watanabe M, Nagai A, Kusumi N, et al. Minimal invasiveness and effectivity of subinguinal microscopic varicocelectomy: a comparative study with retroperitoneal high and laparoscopic approaches. Int J Urol. 2005;12:892-898.

5. Clayman RV, Kavoussi LR, Soper NJ, et al. Laparoscopic nephrectomy: initial case report. J Urol. 1991;146:278-282.

6. Kalloo AN, Singh VK, Jagannath SB, et al. Flexible transgastric peritoneoscopy: a novel approach to diagnostic and therapeutic interventions in the peritoneal cavity. Gastrointest Endosc. 2004; 60:114-117.

7. Swain P. Nephrectomy and natural orifice translumenal endoscopy (NOTES): transvaginal, transgastric, transrectal, and transvesical approaches. J Endourol. 2008;22:811-818.
8. Lima E, Rolanda C, Pêgo JM, et al. Transvesical endoscopic peritoneoscopy: a novel $5 \mathrm{~mm}$ port for intra-abdominal scarless surgery. J Urol. 2006;176:802-805.

9. Lima E, Henriques-Coelho T, Rolanda C, et al. Transvesical thoracoscopy: a natural orifice translumenal endoscopic approach for thoracic surgery. Surg Endosc. 2007;21:854-858.

10. Rolanda C, Lima E, Pêgo JM, et al. Third-generation cholecystectomy by natural orifices: transgastric and transvesical combined approach (with video). Gastrointest Endosc. 2007;65:111-117.

11. Lima E, Rolanda C, Pêgo JM, et al. Third-generation nephrectomy by natural orifice transluminal endoscopic surgery. J Urol. 2007; 178:2648-2654.

12. Lima E, Rolanda C, Osório L, et al. Endoscopic closure of transmural bladder wall perforations. Eur Urol. 2009;56:151-157.

13. Abdulmaaboud MR, Shokeir AA, Farage Y, et al. Treatment of varicocele: a comparative study of conventional open surgery, percutaneous retrograde sclerotherapy, and laparoscopy. Urology. 1998;52:294-300.

14. Thon WF, Gall H, Danz B, et al. Percutaneous sclerotherapy of idiopathic varicocele in childhood: a preliminary report. J Urol. 1989;141:913-915.

15. Kaouk JH, Palmer JS. Single-port laparoscopic surgery: initial experience in children for varicocelectomy. BJU Int. 2008;102:97-99.

16. Zacharakis E, Purkayastha S, Teare J, et al. Natural orifices translumenal endoscopic surgery (NOTES)—who should perform it? Surgery. 2008;144:1-2.

17. Rattner D, Kalloo A, ASGE/SAGES Working Group. ASGE/ SAGES Working Group on Natural Orifice Translumenal Endoscopic Surgery. October 2005. Surg Endosc. 2006;20:329-333.

18. Pai RD, Fong DG, Bundga ME, et al. Transcolonic endoscopic cholecystectomy: a NOTES survival study in a porcine model (with video). Gastrointest Endosc. 2006;64:428-434.

19. Xavier K, Gupta M, Landman J. Transgastric NOTES: current experience and potential implications for urologic applications. J Endourol. 2009;23:737-741.

20. Chang WC, Huang SC, Sheu BC, et al. Transvaginal hysterectomy or laparoscopically assisted vaginal hysterectomy for nonprolapsed uteri. Obstet Gynecol. 2005;106:321-326.

21. Breda G, Silvestre P, Giunta A, et al. Laparoscopic nephrectomy with vaginal delivery of the intact kidney. Eur Urol. 1993;24:116117.

22. Kaouk JH, Haber GP, Goel RK, et al. Pure natural orifice translumenal endoscopic surgery (NOTES) transvaginal nephrectomy. Eur Urol. 2010;57:723-726.

23. Box GN, Bessler M, Clayman RV. Transvaginal access: current experience and potential implications for urologic applications. J Endourol. 2009;23:753-757.

24. Shin EJ, Kalloo AN. Transcolonic NOTES: current experience and potential implications for urologic applications. J Endourol. 2009; 23:743-746.

25. Gettman MT, Blute ML. Transvesical peritoneoscopy: initial clinical evaluation of the bladder as a portal for natural orifice translumenal endoscopic surgery. Mayo Clin Proc. 2007;82:843-845.

26. McGee SM, Routh JC, Pereira CW, et al. Minimal contamination of the human peritoneum after transvesical incision. J Endourol. 2009;23:659-663.

27. Liang JH, Xu CL, Wang LH, et al. Irrigation eliminates smoke formation in laser laparoscopic surgery: ex vivo results. Surg Laparosc Endosc Percutan Tech. 2008;18:391-394.

28. Theisen-Kunde D, Ott V, Brinkmann R, et al. Potential of a new cw $2 \mathrm{~mm}$ laser scalpel for laparoscopic surgery. Med Laser Appl. 2007;22:139-145.

29. Branco F, Pini G, Osório L, et al. Transvesical peritoneoscopy with rigid scope: feasibility study in human male cadaver. Surg Endosc. 2011;25(6):2015-2019.

30. Boylu U, Oommen M, Joshi V, et al. Natural orifice translumenal endoscopic surgery (NOTES) partial nephrectomy in a porcine model. Surg Endosc. 2010;24:485-489. 